\title{
Hydrodynamic Interactions of Two Freely Suspended Droplets in Linear Flow Fields
}

\author{
Li S. Chen and Huan J. Keh ${ }^{1}$ \\ Department of Chemical Engineering, National Taiwan University, Taipei 106-17, Taiwan, Republic of China
}

Received October 27, 1997; accepted April 20, 1998

The hydrodynamic interactions between two fluid droplets freely suspended in an unbounded, immiscible fluid whose velocity at infinity is an arbitrary linear function of position are considered in the quasisteady situation. The droplets may differ in size and in viscosity. It is assumed that the interfacial tensions are sufficiently high so that the droplets retain a spherical shape. The effect of inertial forces on the motion of the fluids inside and outside the droplets is neglected. The relative velocity of the two droplet centers and the force dipole strengths of the two droplets, which are relevant in a calculation of the mean stress in a suspension of many such droplets subjected to bulk deformation, can be represented in terms of several scalar functions of relevant dimensionless parameters. These scalar functions are calculated for various values of the radii, viscosities, and separation distance of the droplets using a boundary collocation technique. The collocation results agree very well with the analytic and numerical results previously obtained for the cases of two solid spheres and two fluid spheres of identical viscosity. $\odot 1998$ Academic Press

Key Words: two-particle hydrodynamic interactions; fluid spheres; linear flow fields; force dipole strength.

\section{INTRODUCTION}

Problems of the hydrodynamic interaction between two solid particles or fluid droplets freely suspended in an unbounded flow field at vanishingly small Reynolds numbers have continued to receive much attention from researchers in the fields of chemical, biomedical, and environmental engineering and science. The majority of the interaction phenomena are fundamental in nature, but enable one to develop rational understanding of many practical properties of a dilute suspension, such as the effective viscosity (1-3), the coagulation rate (4-6), and the shear-induced liquid and particle tracer diffusivities $(7,8)$.

Analytic and numerical solutions for the interaction of two force-free and couple-free solid spheres in a steady linear fluid flow are available in the literature. Lin et al. (9) formulated an exact solution of the Stokes equations for the motion of two neutrally buoyant spheres of arbitrary size and arbitrary orientation with respect to a shear field by using spherical bipolar

\footnotetext{
${ }^{1}$ To whom correspondence should be addressed.
}

coordinates. The numerical results of the particle mobility functions (which can be used to determine the relative trajectories of the particle centers) were obtained for the case of two equally sized spheres freely suspended in a simple shear flow. In this work, the singular behavior of some of these pairinteraction functions at small separations raises the question of convergence in the evaluation of infinite series and Batchelor and Green (10) observed the inconsistency with their asymptotic formulas. The exact solution for the motion of two touching spheres in a shear flow field was developed by Wakiya (11) and Nir and Acrivos (12) through the use of tangent-sphere coordinates. A more detailed analysis of two spheres in a general linear flow field was done by Batchelor and Green (10). They used a method of reflections to obtain the far-field analytic expressions of the relative-velocity and stresslet functions (which are useful in the determination of the bulk stress in a moving suspension of particles) and used a method of lubrication theory for the near-field analytic forms of some of these pair-interaction functions. Yoon and Kim (13) introduced a boundary collocation method (14) which provides accurate information on the pair-interaction functions for the motion of two equally sized spheres in a general linear flow at all separations including the case of touching. In their work, the numerical results for very small separations and the near-field asymptotic expressions analogous to those obtained from lubrication theory are presented. Recently, the boundary collocation method has also been used to obtain solution for the motion of two or more freely suspended, equally sized spheres in planar shear and Poiseuille flow fields (15).

Although the slow motion of two solid particles freely suspended in a linear fluid flow was studied extensively in the past, parallel problems concerning fluid droplets have attracted less attention and are of a higher degree of difficulty because of the fact that the droplet is deformable and an additional flow field inside the droplet must be solved. Using the method of reflections, Zinchenko (16) solved for the motion of two identical force-free fluid spheres in a general linear field and obtained the relative-velocity and stresslet functions as power series in $a / r$ up to $O\left(r^{-6}\right)$, where $a$ is the radius of the droplets and $r$ is their center-to-center distance. In his work, the spherical bipolar coordinate system was also employed to get the 
numerical solutions for some of these pair-interaction functions. Recently, the methods of reflections and of spherical bipolar coordinates are also used by Wang et al. (6) to determine the relative-velocity functions for two differently sized fluid spheres of the same viscosity in a linear flow field. However, the motion of two force-free fluid spheres with different viscosities in a linear flow field has not been examined. Even for the case of two unequally sized solid spheres (or fluid spheres of the same viscosity) freely suspended in a linear flow field, the solution for the pair-interaction functions (or stresslet functions) at an arbitrary separation distance is not yet available in the literature.

The object of the present work is to investigate the hydrodynamic interactions between two arbitrary spherical droplets freely suspended in a general linear flow field. The droplets may differ in radius and have unequal viscosities. The boundary collocation method is used to solve the problem. The detailed discussion of the hydrodynamic interactions between two force-free fluid spheres is presented in Section 2, where we review the relative-velocity and stresslet functions required for the evaluation of the mean stress in a suspension of many such droplets which is subjected to a prescribed bulk deforming motion. In Section 3, the formulation of the boundary collocation method for the slow motion of two force-free fluid spheres in an arbitrary unbounded flow field is given. Collocation equations for three linear undisturbed velocity fields, which are sufficient to result in all the relative-velocity and stresslet functions, are derived, and their solution scheme is furnished. Based on this analysis, the numerical exact solutions of these pair-interaction functions are obtained in Section 4 for various cases. Our results agree well with the existing solutions in the literature.

\section{DEFINITION OF THE PAIR-INTERACTION FUNCTIONS}

Our main purpose in this work is to provide information about the interaction of two force-free fluid spheres immersed in an immiscible moving fluid, with a view to its use for the determination of the bulk stress in a moving suspension of many such droplets. Consider two spherical droplets on which no external force acts in an unbounded fluid of viscosity $\eta$ with the steady linear undisturbed velocity field of arbitrary type,

$$
\mathbf{v}_{\infty}(\mathbf{x})=\mathbf{v}_{\mathrm{o}}+\boldsymbol{\omega} \times \mathbf{x}+\mathbf{E} \cdot \mathbf{x}
$$

where $\mathbf{v}_{\mathrm{o}}, \boldsymbol{\omega}\left(=\frac{1}{2} \boldsymbol{\nabla} \times \mathbf{v}_{\infty}\right)$, and $\mathbf{E}\left(=\frac{1}{2}\left[\boldsymbol{\nabla} \mathbf{v}_{\infty}+\left(\boldsymbol{\nabla} \mathbf{v}_{\infty}\right)^{\mathrm{T}}\right]\right)$ are the constant translational velocity, vorticity vector, and rate-ofstrain dyadic (which is symmetric and traceless), respectively, and $\mathbf{x}$ is the position vector. The droplets may be formed from different fluids and have unequal radii, and they are assumed to be sufficiently small so that interfacial tensions (which are assumed to be fairly large) maintain their spherical shape. The instantaneous position of the center of the first droplet (with radius $a_{1}$ and viscosity $\eta_{1}$ ) is $\mathbf{x}_{1}$ and that of the second droplet (with radius $a_{2}$ and viscosity $\eta_{2}$ ) is $\mathbf{x}_{2}$.

According to the analysis of Batchelor and Green $(1,10)$ for the application of the two-sphere problem to the evaluation of the mean stress in a suspension, it is necessary to determine the velocity of sphere 1 relative to that of sphere 2 and the force dipole strengths of the two spheres. In this work, we use the definition of certain nondimensional scalar functions of the two-sphere hydrodynamic interaction summarized by Batchelor and Green $(1,10)$ and Yoon and Kim (13).

\subsection{The Relative Velocity}

With no loss of generality, the velocity of droplet 2 relative to that of droplet 1 in the undisturbed velocity field given by Eq. [2.1] can be written as

$\mathbf{V}(\mathbf{r})=\boldsymbol{\omega} \times \mathbf{r}+\mathbf{E} \cdot \mathbf{r}-[A \mathbf{e e}+B(\mathbf{I}-\mathbf{e e})] \cdot \mathbf{E} \cdot \mathbf{r}$,

where $\mathbf{I}$ is the unit dyadic and $\mathbf{r}=\mathbf{x}_{2}-\mathbf{x}_{1}=r \mathbf{e}(r=|\mathbf{r}|$ and $\mathbf{e}$ is the unit vector along $\mathbf{r}$ ) is the vector from the center of droplet 1 to the center of droplet 2. The nondimensional scalar quantities $A$ and $B$ are functions only of four dimensionless variables,

$$
\begin{aligned}
\eta_{1}^{*} & =\frac{\eta_{1}}{\eta}, \quad \eta_{2}^{*}=\frac{\eta_{2}}{\eta}, \\
s & =\frac{2 r}{a_{1}+a_{2}}, \quad \lambda=\frac{a_{2}}{a_{1}},
\end{aligned}
$$

and are independent of the values of $\mathbf{E}$ and $\boldsymbol{\omega}$. It follows from the definition of these relative-velocity functions that

$$
\begin{aligned}
& A\left(\eta_{1}^{*}, \eta_{2}^{*}, s, \lambda\right)=A\left(\eta_{2}^{*}, \eta_{1}^{*}, s, \lambda^{-1}\right), \\
& B\left(\eta_{1}^{*}, \eta_{2}^{*}, s, \lambda\right)=B\left(\eta_{2}^{*}, \eta_{1}^{*}, s, \lambda^{-1}\right) .
\end{aligned}
$$

When the two droplets are far apart, each droplet moves approximately as if it were alone in the infinite fluid, that is,

$$
A \rightarrow 0 \quad \text { and } \quad B \rightarrow 0 \quad \text { as } \quad s \rightarrow \infty
$$

When the two spheres are touching, the component of $\mathbf{V}$ parallel to the line of centers must be zero, namely,

$$
A=1 \quad \text { as } \quad s=2 .
$$

\subsection{The Force Dipole Strengths}

The hydrodynamic force dipole strength (stresslet) for a particle with surface $S_{0}$ is defined as 


$$
\mathbf{S}=\int_{S_{\mathrm{o}}}\left[(\mathbf{n} \cdot \boldsymbol{\sigma}) \mathbf{x}-\frac{1}{3}(\mathbf{n} \cdot \boldsymbol{\sigma} \cdot \mathbf{x}) \mathbf{I}-\eta(\mathbf{v n}+\mathbf{n v})\right] d S,
$$

where $\mathbf{v}(\mathbf{x})$ and $\boldsymbol{\sigma}(\mathbf{x})$ are the local fluid velocity and hydrodynamic stress (external to the particle surface), respectively, and $\mathbf{n}$ is the unit outward normal vector for the particle surface. For two force-free fluid spheres in the undisturbed velocity field given by Eq. [2.1], the force dipole strength of droplet $i$, which is a symmetric dyadic with zero trace, can be expressed in the form

$$
\begin{aligned}
\mathbf{S}_{i}(\mathbf{r})= & \frac{4}{3} \pi \eta a_{i}^{3} \frac{2+5 \eta_{i}^{*}}{1+\eta_{i}^{*}}\left\{\left(1+K_{i}\right) \mathbf{E}\right. \\
& +L_{i}\left[(\mathbf{e} \cdot \mathbf{E}) \mathbf{e}+\mathbf{e}(\mathbf{E} \cdot \mathbf{e})-\frac{2}{3}(\mathbf{e} \cdot \mathbf{E} \cdot \mathbf{e}) \mathbf{I}\right] \\
& \left.+M_{i}(\mathbf{e} \cdot \mathbf{E} \cdot \mathbf{e})\left(\mathbf{e} \mathbf{e}-\frac{1}{3} \mathbf{I}\right)\right\} \\
= & \frac{4}{3} \pi \eta a_{i}^{3} \frac{2+5 \eta_{i}^{*}}{1+\eta_{i}^{*}}\left\{\mathbf{E}+K_{i}\left[\mathbf{E}+\frac{1}{2}(\mathbf{e} \cdot \mathbf{E} \cdot \mathbf{e})\right.\right. \\
& \times(\mathbf{e e}+\mathbf{I})-(\mathbf{e} \cdot \mathbf{E}) \mathbf{e}-\mathbf{e}(\mathbf{E} \cdot \mathbf{e})] \\
& +Q_{i}[(\mathbf{e} \cdot \mathbf{E}) \mathbf{e}+\mathbf{e}(\mathbf{E} \cdot \mathbf{e})-2(\mathbf{e} \cdot \mathbf{E} \cdot \mathbf{e}) \mathbf{e e}] \\
& \left.+\frac{1}{2} P_{i}(\mathbf{e} \cdot \mathbf{E} \cdot \mathbf{e})(3 \mathbf{e e}-\mathbf{I})\right\},
\end{aligned}
$$

where $i=1$ or 2 . The dimensionless scalar quantities $K_{i}, Q_{i}$, and $P_{i}\left(L_{i}=Q_{i}-K_{i}, \quad M_{i}=\left(3 P_{i}-3 K_{i}-4 L_{i}\right) / 2\right)$ are functions only of variables $\eta_{1}^{*}, \eta_{2}^{*}, s$, and $\lambda$ as defined in Eqs. [2.3] and [2.4], and are independent of the bulk flow. The expression of $\mathbf{S}_{i}(\mathbf{r})$ in terms of the stresslet functions $K_{i}, L_{i}$, and $M_{i}$ was employed by Batchelor and Green $(1,10)$, while that in terms of $K_{i}, Q_{i}$, and $P_{i}$ was used by Yoon and Kim (13). It can be shown that

$$
\begin{aligned}
& K_{1}\left(\eta_{1}^{*}, \eta_{2}^{*}, s, \lambda\right)=K_{2}\left(\eta_{2}^{*}, \eta_{1}^{*}, s, \lambda^{-1}\right), \\
& Q_{1}\left(\eta_{1}^{*}, \eta_{2}^{*}, s, \lambda\right)=Q_{2}\left(\eta_{2}^{*}, \eta_{1}^{*}, s, \lambda^{-1}\right), \\
& P_{1}\left(\eta_{1}^{*}, \eta_{2}^{*}, s, \lambda\right)=P_{2}\left(\eta_{2}^{*}, \eta_{1}^{*}, s, \lambda^{-1}\right),
\end{aligned}
$$

and

$$
K_{i} \rightarrow 0, \quad Q_{i} \rightarrow 0, \quad \text { and } \quad P_{i} \rightarrow 0 \quad \text { as } \quad s \rightarrow \infty \text {. }
$$

Note that the only instantaneous consequence of the uniform vorticity at infinity is to contribute a rigid-body rotation with angular velocity $\boldsymbol{\omega}$ to the whole system. It is for this reason that the quantities $\mathbf{V}(\mathbf{r})-\boldsymbol{\omega} \times \mathbf{r}$ and $\mathbf{S}_{i}(\mathbf{r})$ must be linear and

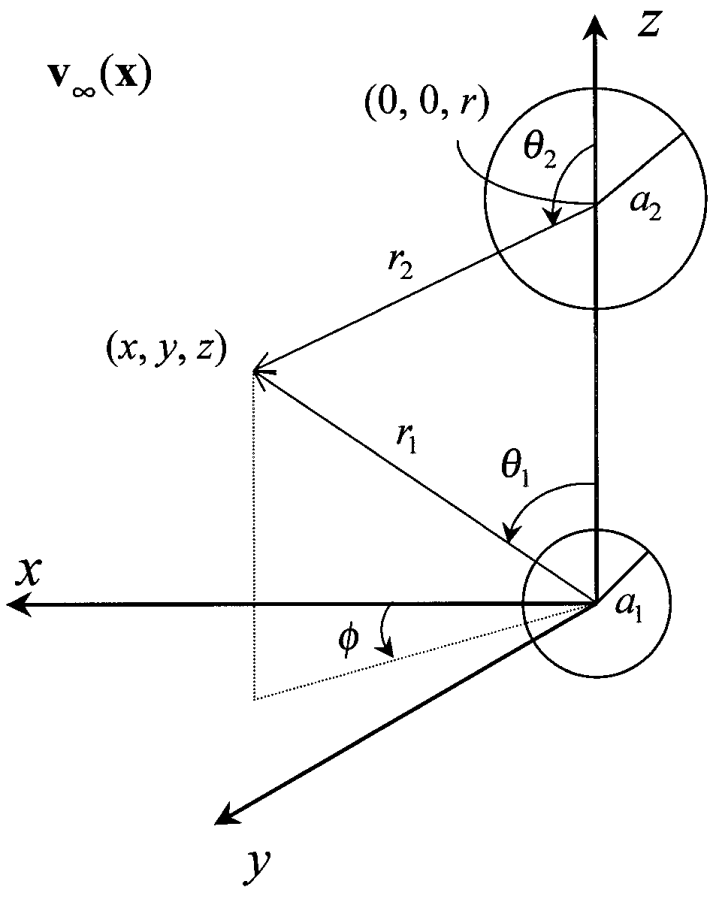

FIG. 1. Geometrical sketch of two spherical droplets in an immiscible fluid with an undisturbed velocity field $\mathbf{v}_{\infty}(\mathbf{x})$.

homogeneous in $\mathbf{E}$ and that we are able to find forms for the scalar functions describing instantaneous kinematic and dynamic properties of the two-sphere interactions which are independent of $\boldsymbol{\omega}$. Equations [2.5] and [2.9] show that the pair-interaction functions $A, B, K_{i}, Q_{i}$, and $P_{i}$ are to be determined for $2 \leq s<\infty$ and $1 \leq \lambda<\infty$.

\section{BOUNDARY COLLOCATION ANALYSIS}

We consider the slow motion of two neutrally buoyant spherical droplets in an unbounded, immiscible fluid with an undisturbed velocity field $\mathbf{v}_{\infty}(\mathbf{x})$, as shown in Fig. 1. For convenience, the rectangular coordinate system $(x, y, z)$ with principal unit vectors $\mathbf{e}_{x}, \mathbf{e}_{y}$, and $\mathbf{e}_{z}$ is established such that the center of the first sphere is at the origin. The center of the other droplet is positioned on the positive $z$-axis with a distance $r$ from the origin (so that $\mathbf{e}=\mathbf{e}_{z}$ ). Assume that the Reynolds numbers are vanishingly small and that the problem can be viewed quasisteady. Hence, the fluid velocities inside and outside the droplets are governed by the Stokes equations

$$
\begin{aligned}
\eta_{i} \boldsymbol{\nabla}^{2} \mathbf{v}_{i}-\boldsymbol{\nabla} p_{i} & =\mathbf{0}, \\
\boldsymbol{\nabla} \cdot \mathbf{v}_{i} & =0, \\
\eta \nabla^{2} \mathbf{v}-\nabla p & =\mathbf{0}, \\
\boldsymbol{\nabla} \cdot \mathbf{v} & =0,
\end{aligned}
$$

for $i=1$ or 2 . Here, $\mathbf{v}_{i}(\mathbf{x})$ and $\mathbf{v}(\mathbf{x})$ are the velocity fields for the 
flow inside droplet $i$ and for the external flow, respectively; $p_{i}(\mathbf{x})$ and $p(\mathbf{x})$ are the corresponding dynamic pressure distributions.

The boundary conditions for the fluid velocities at the droplet surfaces and at infinity are

$$
\begin{gathered}
r_{i}=a_{i}: \quad \mathbf{v}=\mathbf{v}_{i}, \\
\mathbf{e}_{r i} \cdot\left(\mathbf{v}-\mathbf{U}_{i}\right)=0, \\
\left(\mathbf{I}-\mathbf{e}_{r i} \mathbf{e}_{r i}\right) \mathbf{e}_{r i}:\left(\boldsymbol{\tau}-\boldsymbol{\tau}_{i}\right)=\mathbf{0}, \\
\left(x^{2}+y^{2}+z^{2}\right) \rightarrow \infty: \quad \mathbf{v} \rightarrow \mathbf{v}_{\infty}(\mathbf{x}) .
\end{gathered}
$$

Here, $\left(r_{i}, \theta_{i}, \phi\right)$ are the spherical coordinates measured from the center of droplet $i ; \mathbf{e}_{r i}$ is the radial unit vector in the spherical coordinates; and $\mathbf{U}_{i}$, equal to $U_{i x} \mathbf{e}_{x}+U_{i y} \mathbf{e}_{y}+U_{i z} \mathbf{e}_{z}$, is the translational velocity of droplet $i$ to be determined by the requirement that the hydrodynamic force on it is zero. The deviatoric stress tensors for the external flow and the flow inside droplet $i$ can be expressed as

$$
\boldsymbol{\tau}=\eta\left[\boldsymbol{\nabla} \mathbf{v}+(\boldsymbol{\nabla} \mathbf{v})^{\mathrm{T}}\right]
$$

and

$$
\boldsymbol{\tau}_{i}=\eta_{i}\left[\boldsymbol{\nabla} \mathbf{v}_{i}+\left(\boldsymbol{\nabla} \mathbf{v}_{i}\right)^{\mathrm{T}}\right]
$$

respectively. (In Eq. [2.7], $\boldsymbol{\sigma}=-p \mathbf{I}+\boldsymbol{\tau}$.) The normal components of the normal stress vectors at the surface of droplet $i$ have a discontinuity which is proportional to the surface tension. Presently we assume that the droplets remain to be spherical and shall not use this boundary condition.

The general solution to Eq. [3.1] is

$$
\begin{aligned}
\mathbf{v}= & \mathbf{v}_{\infty}(\mathbf{x})+\sum_{j=1}^{2} \sum_{n=1}^{\infty}\left[\boldsymbol{\nabla} \times\left(\mathbf{r}_{j} \chi_{-n-1}^{(j)}\right)+\boldsymbol{\nabla} \Phi_{-n-1}^{(j)}\right. \\
& \left.-\frac{n-2}{2 \eta n(2 n-1)} r_{j}^{2} \nabla p_{-n-1}^{(j)}+\frac{n+1}{\eta n(2 n-1)} \mathbf{r}_{j} p_{-n-1}^{(j)}\right],
\end{aligned}
$$

$$
\begin{aligned}
\mathbf{v}_{i}= & \mathbf{v}_{\infty}\left(\mathbf{x}_{i}\right)+\sum_{n=1}^{\infty}\left[\boldsymbol{\nabla} \times\left(\mathbf{r}_{i} \chi_{n}^{(i)}\right)+\nabla \Phi_{\mathrm{n}}^{(\mathrm{i})}\right. \\
& +\frac{n+3}{2 \eta_{i}(n+1)(2 n+3)} r_{i}^{2} \nabla p_{n}^{(i)} \\
& \left.-\frac{n}{\eta_{i}(n+1)(2 n+3)} \mathbf{r}_{i} p_{n}^{(i)}\right]
\end{aligned}
$$

where $\mathbf{x}_{i}$ is the position of the center of droplet $i, \chi_{n}^{(i)}, \Phi_{\mathrm{n}}^{(\mathrm{i})}$, and $p_{n}^{(i)}$ are solid spherical harmonic functions of order $n$ which depend on the spherical coordinates $\left(r_{i}, \theta_{i}, \phi\right)$ originating at the center of droplet $i$, and $\mathbf{r}_{i}=r_{i} \mathbf{e}_{r i}$. These harmonic functions can be expressed as

$$
\begin{aligned}
& {\left[\begin{array}{l}
\chi_{-n-1}^{(j)} \\
\Phi_{-n-1}^{(j)} \\
p_{-n-1}^{(j)}
\end{array}\right]=} r_{j}^{-n-1} \sum_{m=0}^{n} P_{n}^{m}\left(\mu_{j}\right) \\
& \times\left\{\left[\begin{array}{l}
A_{j m n} \\
C_{j m n} \\
E_{j m n}
\end{array}\right] \cos (m \phi)+\left[\begin{array}{c}
B_{j m n} \\
D_{j m n} \\
F_{j m n}
\end{array}\right] \sin (m \phi)\right\}, \quad[3.5 \\
& {\left[\begin{array}{l}
\chi_{n}^{(i)} \\
\Phi_{\mathrm{n}}^{(\mathrm{i})} \\
\mathrm{p}_{\mathrm{n}}^{(\mathrm{i})}
\end{array}=\mathrm{r}_{\mathrm{i}}^{\mathrm{n}} \sum_{\mathrm{m}=0}^{n} P_{n}^{m}\left(\mu_{i}\right)\left\{\left[\begin{array}{l}
\bar{A}_{i m n} \\
\bar{C}_{i m n} \\
\bar{E}_{i m n}
\end{array}\right] \cos (m \phi)+\left[\begin{array}{c}
\bar{B}_{i m n} \\
\bar{D}_{i m n} \\
\bar{F}_{i m n}
\end{array}\right] \sin (m \phi)\right\},\right.}
\end{aligned}
$$

where $P_{n}^{m}\left(\mu_{i}\right)$ is the associated Legendre function of order $m$ and degree $n$ and $\mu_{i}$ is used to denote $\cos \theta_{i}$ for brevity. The requirement of finite fluid velocity in the interior of each droplet and the boundary condition [3.2d] are immediately satisfied by a solution of this form. The unknown coefficients $A_{j m n}, B_{j m n}, \ldots, F_{j m n}$ and $\bar{A}_{i m n}, \bar{B}_{i m n}, \ldots, \bar{F}_{i m n}$ for a given undisturbed velocity field $\mathbf{v}_{\infty}(\mathbf{x})$ are to be determined from boundary conditions $[3.2 \mathrm{a}-\mathrm{c}]$ in terms of the droplet velocities $\mathbf{U}_{i}$ using the boundary collocation technique $(14,17)$. In the construction of solution [3.4], the superposition of Lamb's (18) general solution to the Stokes equations as written from two different origins can be used because of the linearity of the system.

The stress vector acting across the surface of droplet $i$ is

$$
\begin{aligned}
\mathbf{e}_{r i} \cdot \boldsymbol{\sigma}= & \frac{\eta}{r_{i}} \sum_{n=1}^{\infty}\left[-(n+2) \boldsymbol{\nabla} \times\left(\mathbf{r}_{i} \chi_{-(n+1)}^{(i)}\right)\right. \\
& -2(n+2) \boldsymbol{\nabla} \Phi_{-(n+1)}^{(i)}+\frac{n^{2}-1}{\eta n(2 n-1)} r_{i}^{2} \boldsymbol{\nabla} p_{-(n+1)}^{(i)} \\
& \left.-\frac{2 n^{2}+1}{\eta n(2 n-1)} \mathbf{r}_{i} p_{-(n+1)}^{(i)}\right]
\end{aligned}
$$

The drag force exerted by the external fluid on the droplet is obtained by integrating this stress around the droplet surface, with the simple result (19)

$$
\mathbf{F}_{i}=-4 \pi \nabla\left[r_{i}^{3} p_{-2}^{(i)}\right]
$$

That is, only a lowest-order external solid spherical harmonic function contributes to the hydrodynamic force on each droplet. Substitution of Eq. [3.5a] into Eq. [3.7] leads to

$$
\mathbf{F}_{i}=-4 \pi\left(E_{i 11} \mathbf{e}_{x}+F_{i 11} \mathbf{e}_{y}+E_{i 01} \mathbf{e}_{z}\right)
$$


Because the droplets are force-free, the net force exerted by the external fluid on the surface of each droplet must vanish, and we have

$$
E_{i 01}=E_{i 11}=F_{i 11}=0, \quad i=1,2 .
$$

The velocities $\mathbf{U}_{i}$ of the two droplets (six components in total) for a given undisturbed flow field can be obtained by solving Eqs. [3.9] (six equations) simultaneously.

The basic object of this work is to examine the dependence of the scalar functions $A, B, K_{i}, Q_{i}$, and $P_{i}$, defined by expressions [2.2] and [2.8] for two spherical droplets, on the parameters $\eta_{1}^{*}, \eta_{2}^{*}, s$, and $\lambda$. Because these pair-interaction functions are independent of the undisturbed velocity $\mathbf{v}_{\infty}(\mathbf{x})$ given by Eq. [2.1], calculations made for particular ambient flow fields can be used as a source of information about the values of these scalar functions. In the following subsections, three simple velocity fields of $\mathbf{v}_{\infty}(\mathbf{x})$ will be considered, which suffices to yield the results of all the pairinteraction functions.

\subsection{Two Droplets in a Two-Dimensional Straining Flow}

In this subsection, we consider the motion of two fluid spheres in the velocity field

$$
\mathbf{v}_{\infty}(\mathbf{x})=\kappa\left(x \mathbf{e}_{x}-y \mathbf{e}_{y}\right)
$$

or

$$
\mathbf{E}=\kappa\left(\mathbf{e}_{x} \mathbf{e}_{x}-\mathbf{e}_{y} \mathbf{e}_{y}\right) .
$$

In this two-dimensional pure straining flow, the $z$-axis, on which the two droplets are located, is a line of stagnation points. Thus, the droplet velocities $\mathbf{U}_{i}=\mathbf{0}$ and the coefficients $A_{j m n}, D_{j m n}, F_{j m n}, \bar{A}_{i m n}, \bar{D}_{i m n}$, and $\bar{F}_{i m n}$ in Eq. [3.5] are all zero. Substituting Eq. [3.10b] into Eq. [2.8], we obtain

$$
\mathbf{S}_{i}=\frac{4}{3} \pi \eta a_{i}^{3} \frac{2+5 \eta_{i}^{*}}{1+\eta_{i}^{*}} \kappa\left(1+K_{i}\right)\left(\mathbf{e}_{x} \mathbf{e}_{x}-\mathbf{e}_{y} \mathbf{e}_{y}\right)
$$

Application of the boundary conditions [3.2a-c] at the droplet surfaces with $\mathbf{U}_{i}=\mathbf{0}$ to the general solution [3.4] after the substitution of the undisturbed velocity [3.10a] leads to

$$
\begin{aligned}
& H_{i 2}^{(1)}\left(\mu_{i}\right)=-\kappa a_{i}\left(1-\mu_{i}^{2}\right), \\
& H_{i 2}^{(2)}\left(\mu_{i}\right)=-\kappa a_{i} \mu_{i}\left(1-\mu_{i}^{2}\right)^{1 / 2}, \\
& H_{i 2}^{(3)}\left(\mu_{i}\right)=\kappa a_{i}\left(1-\mu_{i}^{2}\right)^{1 / 2}, \\
& H_{i 2}^{(4)}\left(\mu_{i}\right)=-\kappa a_{i}\left(1-\mu_{i}^{2}\right),
\end{aligned}
$$

$$
\begin{aligned}
& H_{i 2}^{(5)}\left(\mu_{i}\right)=-2 \kappa a_{i} \mu_{i}\left(1-\mu_{i}^{2}\right)^{1 / 2}, \\
& H_{i 2}^{(6)}\left(\mu_{i}\right)=2 \kappa a_{i}\left(1-\mu_{i}^{2}\right)^{1 / 2},
\end{aligned}
$$

where $i=1$ or 2 . In Eq. [3.12], the functions $H_{i 2}^{(k)}\left(\mu_{i}\right)$ with $k=1,2, \ldots, 6$ are infinite series defined by Eqs. [B1]-[B6] in Appendix B and the dependence on $\phi$ factors out. To satisfy boundary conditions [3.12a-f] exactly along the entire surface of each spherical droplet would require the solution of the entire infinite arrays of unknown constants $B_{j 2 n}, C_{j 2 n}, E_{j 2 n}$, $\bar{B}_{i 2 n}, \bar{C}_{i 2 n}$, and $\bar{E}_{i 2 n}$ (all constants with $m \neq 2$ are equal to zero) in Eq. [3.5]. However, the boundary collocation method $(14,17)$ allows one to truncate the infinite series into finite ones with $N$ terms, and then to enforce the boundary conditions at $N$ discrete points (values of $\theta_{i}$ or $\mu_{i}$ ) on the generating arc of each sphere. As a result, for our two-droplet problem, this will lead to a set of $12 \mathrm{~N}$ simultaneous linear algebraic equations in the truncated from of Eq. [3.12] for the $12 N$ unknown constants of the truncated solution [3.4]. These equations can be solved by any standard matrix reduction technique. In general, the larger the value of $N$, the more accurate the result will be by this truncation method. Naturally, the truncation error vanishes as $N \rightarrow \infty$.

Substituting the external fluid velocity and stress expressed by Eqs. [3.4a] and [3.6] with the undisturbed velocity field given by Eq. [3.10a] into the force dipole strength expression [2.7] for droplet $i$ and performing the integration with respect to the azimuthal angle $\phi\left(d S=-a_{i}^{2} d \mu_{i} d \phi\right)$, we obtain

$$
\begin{aligned}
\mathbf{S}_{i}= & \left(\mathbf{e}_{x} \mathbf{e}_{x}-\mathbf{e}_{y} \mathbf{e}_{y}\right) \pi \eta a_{i}^{2} \int_{-1}^{1} \sum_{j=1}^{2} \sum_{n=2}^{\infty}\left[\mu_{i}^{2} G_{j i 2 n}^{(1)}\left(a_{i}, \mu_{i}\right)\right. \\
& -\frac{3}{2} \mu_{i}\left(1-\mu_{i}^{2}\right)^{1 / 2} G_{j i 2 n}^{(2)}\left(a_{i}, \mu_{i}\right)+\frac{3}{2}\left(1-\mu_{i}^{2}\right)^{1 / 2} \\
& \times G_{j i 2 n}^{(3)}\left(a_{i}, \mu_{i}\right)+\left(1-\mu_{i}^{2}\right) G_{j i 2 n}^{(4)}\left(a_{i}, \mu_{i}\right) \\
& +\frac{1}{2} \mu_{i}\left(1-\mu_{i}^{2}\right)^{1 / 2} G_{j i 2 n}^{(5)}\left(a_{i}, \mu_{i}\right)-\frac{1}{2}\left(1-\mu_{i}^{2}\right)^{1 / 2} \\
& \times G_{j i 2 n}^{(6)}\left(a_{i}, \mu_{i}\right)+\frac{1}{2} \mu_{i}\left(1-\mu_{i}^{2}\right)^{1 / 2} G_{j i 2 n}^{(7)}\left(a_{i}, \mu_{i}\right) \\
& \left.-\frac{1}{2}\left(1-\mu_{i}^{2}\right) G_{j i 2 n}^{(8)}\left(a_{i}, \mu_{i}\right)\right] d \mu_{i},
\end{aligned}
$$

where $i=1$ or 2. In Eq. [3.13], the definition of the functions $G_{j i 2 n}^{(l)}\left(r_{i}, \mu_{i}\right)$ with $l=1,2, \ldots, 8$ is given by Eq. [B7] in Appendix B. After the coefficients $B_{j 2 n}, C_{j 2 n}$, and $E_{j 2 n}$ are determined, Eq. [3.13] can be numerically integrated and compared with Eq. [3.11] to yield the scalar functions $K_{i}$ for given values of $\eta_{1}^{*}, \eta_{2}^{*}, s$, and $\lambda$. 


\subsection{Two Droplets in a Unidirectional Shear Flow}

We now consider the motion of two spherical droplets in the velocity field

$$
\mathbf{v}_{\infty}(\mathbf{x})=2 \kappa z \mathbf{e}_{x}
$$

or

$$
\begin{aligned}
& \mathbf{E}=\kappa\left(\mathbf{e}_{x} \mathbf{e}_{z}+\mathbf{e}_{z} \mathbf{e}_{x}\right), \\
& \boldsymbol{\omega}=\kappa \mathbf{e}_{y} .
\end{aligned}
$$

In this simple shear flow, the droplet velocities $\mathbf{U}_{i}=U_{i x} \mathbf{e}_{x}$, and again the coefficients $A_{j m n}, D_{j m n}, F_{j m n}, \bar{A}_{i m n}, \bar{D}_{i m n}$, and $\bar{F}_{i m n}$ in Eq. [3.5] vanish. Substitution of Eqs. [3.14b, c] into Eqs. [2.2] and [2.8] gives

$$
\begin{aligned}
\mathbf{V} & =(2-B) \kappa r \mathbf{e}_{x} \\
\mathbf{S}_{i} & =\frac{4}{3} \pi \eta a_{i}^{3} \frac{2+5 \eta_{i}^{*}}{1+\eta_{i}^{*}} \kappa\left(1+Q_{i}\right)\left(\mathbf{e}_{x} \mathbf{e}_{z}+\mathbf{e}_{z} \mathbf{e}_{x}\right) .
\end{aligned}
$$

Application of Eqs. [3.2a-c] with $U_{i y}=U_{i z}=0$ to Eq. [3.4] after the substitution of Eq. [3.14a] results in

$H_{i 1}^{(1)}\left(\mu_{i}\right)=-2 \kappa a_{i} \mu_{i}\left(1-\mu_{i}^{2}\right)^{1 / 2}$,

$H_{i 1}^{(2)}\left(\mu_{i}\right)=-2 \kappa a_{i} \mu_{i}^{2}$,

$H_{i 1}^{(3)}\left(\mu_{i}\right)=2 \kappa a_{i} \mu_{i}$,

$H_{i 1}^{(4)}\left(\mu_{i}\right)=\left[U_{i x}-2(i-1) \kappa r-2 \kappa a_{i} \mu_{i}\right]\left(1-\mu_{i}^{2}\right)^{1 / 2}$,

$H_{i 1}^{(5)}\left(\mu_{i}\right)=2 \kappa a_{i}\left(1-2 \mu_{i}^{2}\right)$,

$H_{i 1}^{(6)}\left(\mu_{i}\right)=2 \kappa a_{i} \mu_{i}$,

where $i=1$ or 2 , and the functions $H_{i 1}^{(k)}\left(\mu_{i}\right)$ with $k=1$, $2, \ldots, 6$ are infinite series defined by Eqs. [B1]-[B6]. Now, the boundary collocation technique described in the previous subsection can be used. The infinite series in boundary conditions [3.17a-f] are truncated after $N$ terms, and these truncated conditions are applied at $N$ discrete points (values of $\theta_{i}$ ) along the droplet surfaces. This generates a system of $12 \mathrm{~N}$ linear algebraic equations for $12 N$ unknown coefficients $B_{j 1 n}, C_{j 1 n}$, $E_{j 1 n}, \bar{B}_{i 1 n}, \bar{C}_{i 1 n}$, and $\bar{E}_{i 1 n}$ (coefficients with $m \neq 1$ are all zero) in Eq. [3.5]. After these coefficients are determined, the solutions for the fluid velocity field and the droplet velocities are completely known.

Substituting Eqs. [3.4a] and [3.6] with Eq. [3.14a] into Eq. [2.7] for the $i$ th droplet, we have

$$
\begin{aligned}
\mathbf{S}_{i}= & \left(\mathbf{e}_{x} \mathbf{e}_{z}+\mathbf{e}_{z} \mathbf{e}_{x}\right) \pi \eta a_{i}^{2} \int_{-1}^{1} \sum_{j=1}^{2} \sum_{n=1}^{\infty}\left[-\mu_{i}\left(1-\mu_{i}^{2}\right)^{-1 / 2}\right. \\
& \times\left(1-2 \mu_{i}^{2}\right) G_{j i 1 n}^{(1)}\left(a_{i}, \mu_{i}\right)+\left(1-3 \mu_{i}^{2}\right) G_{j i 1 n}^{(2)}\left(a_{i}, \mu_{i}\right) \\
& +2 \mu_{i} G_{j i 1 n}^{(3)}\left(a_{i}, \mu_{i}\right)+2 \mu_{i}\left(1-\mu_{i}^{2}\right)^{1 / 2} G_{j i 1 n}^{(4)}\left(a_{i}, \mu_{i}\right) \\
& +\mu_{i}^{2} G_{j i 1 n}^{(5)}\left(a_{i}, \mu_{i}\right)-\mu_{i} G_{j i 1 n}^{(6)}\left(a_{i}, \mu_{i}\right)+\mu_{i}^{2} G_{j i 1 n}^{(7)}\left(a_{i}, \mu_{i}\right) \\
& \left.-\mu_{i}\left(1-\mu_{i}^{2}\right)^{1 / 2} G_{j i 1 n}^{(8)}\left(a_{i}, \mu_{i}\right)\right] d \mu_{i},
\end{aligned}
$$

where $i=1$ or 2 , and the functions $G_{j i 1 n}^{(l)}\left(r_{i}, \mu_{i}\right)$ with $l=1$, $2, \ldots, 8$ are defined by Eq. [B7]. After the coefficients $B_{j 1 n}$, $C_{j 1 n}$, and $E_{j 1 n}$ are solved, we can calculate the relative velocity $\mathbf{V}$ and the force dipole strength $\mathbf{S}_{i}$ (using Eq. [3.18]) of the two droplets. A comparison between these results and those given by Eqs. [3.15] and [3.16] yields the scalar functions $B$ and $Q_{i}$ for given values of $\eta_{1}^{*}, \eta_{2}^{*}, s$, and $\lambda$.

\subsection{Two Droplets in a Three-Dimensional Straining Flow}

In this subsection we consider the motion of two spherical droplets in the velocity field

$$
\mathbf{v}_{\infty}(\mathbf{x})=\kappa\left(-\frac{1}{2} x \mathbf{e}_{x}-\frac{1}{2} y \mathbf{e}_{y}+z \mathbf{e}_{z}\right)
$$

or

$$
\begin{aligned}
& \mathbf{E}=\kappa\left(-\frac{1}{2} \mathbf{e}_{x} \mathbf{e}_{x}-\frac{1}{2} \mathbf{e}_{y} \mathbf{e}_{y}+\mathbf{e}_{z} \mathbf{e}_{z}\right), \\
& \boldsymbol{\omega}=\mathbf{0} .
\end{aligned}
$$

In this axisymmetric pure straining flow, the droplet velocities $\underline{\mathbf{U}}_{i}=U_{i z} \mathbf{e}_{z}$ and the coefficients $A_{j m n}, B_{j m n}, D_{j m n}, F_{j m n}, \bar{A}_{i m n}$, $\bar{B}_{i m n}, \bar{D}_{i m n}$, and $\bar{F}_{i m n}$ in Eq. [3.5] are all zero. Substituting Eqs. [3.19b, c] into Eqs. [2.2] and [2.8], we have

$\mathbf{V}=(1-A) \kappa r \mathbf{e}_{z}$,

$\mathbf{S}_{i}=\frac{4}{3} \pi \eta a_{i}^{3} \frac{2+5 \eta_{i}^{*}}{1+\eta_{i}^{*}} \kappa\left(1+P_{i}\right)\left(-\frac{1}{2} \mathbf{e}_{x} \mathbf{e}_{x}-\frac{1}{2} \mathbf{e}_{y} \mathbf{e}_{y}+\mathbf{e}_{z} \mathbf{e}_{z}\right)$.

Application of Eqs. [3.2a-c] with $U_{i x}=U_{i y}=0$ to Eq. [3.4] after the substitution of Eq. [3.19a] yields

$H_{i 0}^{(1)}\left(\mu_{i}\right)=\frac{1}{2} \kappa a_{i}\left(1-3 \mu_{i}^{2}\right)$

$H_{i 0}^{(2)}\left(\mu_{i}\right)=\frac{3}{2} \kappa a_{i} \mu_{i}\left(1-\mu_{i}^{2}\right)^{1 / 2}$, 


$$
\begin{aligned}
& H_{i 0}^{(4)}\left(\mu_{i}\right)=\left[U_{i z}-(i-1) \kappa r\right] \mu_{i}+\frac{1}{2} \kappa a_{i}\left(1-3 \mu_{i}^{2}\right) \\
& H_{i 0}^{(5)}\left(\mu_{i}\right)=3 \kappa a_{i} \mu_{i}\left(1-\mu_{i}^{2}\right)^{1 / 2}
\end{aligned}
$$

where $i=1$ or 2 , and the definitions of infinite-series functions $H_{i 0}^{(k)}\left(\mu_{i}\right)$ with $k=1,2,4$, and 5 are given by Eqs. [B1], [B2], [B4], and [B5]. As described in the previous subsections, we can use the boundary collocation technique to solve the first $N$ sets of the coefficients $C_{j 0 n}, E_{j 0 n}, \bar{C}_{i 0 n}$, and $\bar{E}_{i 0 n}$ (all coefficients with $m \neq 0$ disappear) in Eq. [3.5] and then to obtain the solutions for the flow field and the droplet velocities.

Substitution of Eqs. [3.4a] and [3.6] with Eq. [3.19a] into Eq. [2.7] for droplet $i$ leads to

$$
\begin{aligned}
\mathbf{S}_{i}= & \left(-\frac{1}{2} \mathbf{e}_{x} \mathbf{e}_{x}-\frac{1}{2} \mathbf{e}_{y} \mathbf{e}_{y}+\mathbf{e}_{z} \mathbf{e}_{z}\right) \pi \eta a_{i}^{2} \int_{-1}^{1} \sum_{j=1}^{2} \sum_{n=1}^{\infty}\left[-4 \mu_{i}^{2}\right. \\
& \times G_{j i 0 n}^{(1)}\left(a_{i}, \mu_{i}\right)+6 \mu_{i}\left(1-\mu_{i}^{2}\right)^{1 / 2} G_{j i o n}^{(2)}\left(a_{i}, \mu_{i}\right) \\
& -\frac{4}{3}\left(1-3 \mu_{i}^{2}\right) G_{j i 0 n}^{(4)}\left(a_{i}, \mu_{i}\right)-2 \mu_{i}\left(1-\mu_{i}^{2}\right)^{1 / 2} \\
& \times G_{j i 0 n}^{(5)}\left(a_{i}, \mu_{i}\right)-2 \mu_{i}\left(1-\mu_{i}^{2}\right)^{1 / 2} G_{j i i n}^{(7)}\left(a_{i}, \mu_{i}\right) \\
& \left.+\frac{2}{3}\left(1-3 \mu_{i}^{2}\right) G_{j i 0 n}^{(8)}\left(a_{i}, \mu_{i}\right)\right] d \mu_{i},
\end{aligned}
$$

where $i=1$ or 2 , and the functions $G_{j i 0 n}^{(l)}\left(r_{i}, \mu_{i}\right)$ with $l=1$, $2, \ldots, 8$ are defined by Eq. [B7]. After the coefficients $C_{j 0 n}$ and $E_{j 0 n}$ are solved, we can calculate the relative velocity $\mathbf{V}$ and the force dipole strength $\mathbf{S}_{i}$ (using Eq. [3.23]) of the two droplets and compare the results with Eqs. [3.20] and [3.21] to obtain the scalar functions $A$ and $P_{i}$.

\section{RESULTS AND DISCUSSION}

In this section, the numerical results of the dimensionless pair-interaction functions $A, B, K_{i}, Q_{i}$, and $P_{i}$ defined in Eqs. [2.2] and [2.8], obtained by using the boundary collocation method described in the previous section, will be presented. The details of the collocation scheme used for this work were given by Keh and Tseng (17). The Crout method (20) has been employed to solve the matrix equations [3.12], [3.17], and [3.22], and the method of Gauss-Legendre quadrature with 200 zeros is used to perform the integration in Eqs. [3.13], [3.18], and [3.23]. A DEC 3000/600 workstation was used to accomplish the calculations. The accuracy and convergence behavior of the truncation depends principally upon the relative spacing of the droplets. For the difficult case of two touching droplets, the number of collocation points $N=300$ is sufficiently large to achieve satisfactory convergence.
TABLE 1

The Scalar Functions $B, K, Q$, and $P$ of Two Identical Touching D roplets at Various Values of the R elative V iscosity $\eta^{*}$

\begin{tabular}{lllll}
\hline$\eta^{*}$ & \multicolumn{1}{c}{$B$} & \multicolumn{1}{c}{$K$} & $Q$ & \multicolumn{1}{c}{$P$} \\
\hline 0 & 0 & 0 & -0.0985 & 0.99 \\
0.1 & 0.0103 & -0.0060 & -0.0941 & 1.13 \\
0.2 & 0.0195 & -0.0109 & -0.0907 & 1.12 \\
0.5 & 0.0420 & $-0.0209 *$ & -0.0828 & 1.052 \\
1 & 0.0686 & $-0.0299^{*}$ & -0.0735 & 0.994 \\
2 & 0.1014 & $-0.0377^{*}$ & -0.0608 & 0.951 \\
5 & 0.1489 & $-0.0439^{*}$ & -0.0383 & 0.924 \\
10 & 0.1844 & $-0.0459^{*}$ & -0.0180 & 0.9160 \\
$\infty$ & 0.4060 & $-0.0472 *$ & 0.1454 & 0.9105 \\
\hline
\end{tabular}

* Values also obtained by Zinchenko (16).

For the case of two identical droplets $\left(a_{1}=a_{2}=a\right.$, $\eta_{1}^{*}=\eta_{2}^{*}=\eta^{*}, K_{1}=K_{2}=K, Q_{1}=Q_{2}=Q, P_{1}=P_{2}=P$ ) in a linear fluid flow, our collocation solutions of the pair-interaction functions $A, B, K, Q$, and $P$ converge to at least four digits after the decimal point at various values of $\eta^{*}$ and $s$ in the whole possible range of $0 \leq \eta^{*}<\infty$ and $0 \leq 2 / s \leq 1$ (with some exceptions of the function $P$ at $s=2$ ). These collocation results are in excellent agreement with the corresponding results obtained by Zinchenko (16) using the methods of reflections and of spherical bipolar coordinates. Because of the limitation of the methods, Zinchenko could not calculate the scalar functions $A, B, Q$, and $P$ for the particular case of two touching droplets $(s=2)$. In Table 1 , we list the results of functions $B, K, Q$, and $P$ for two touching droplets with various values of the relative viscosity $\eta^{*}(A=1$ in this case).

In Table 2 we list the numerical solutions of the scalar functions $A, Q_{1}, P_{1}, Q_{2}$, and $P_{2}$ for the limiting case of two gas bubbles in liquid $\left(\eta_{1}^{*}=\eta_{2}^{*} \rightarrow 0\right)$ at various relative radii and spacings. It can be shown that the scalar functions $B, K_{1}$, and $K_{2}$ for this limiting case are always zero (16). The values of the function $A$ in Table 2 agree well with the corresponding plots given by Wang et al. (6).

The collocation results of the scalar functions for the other limiting case of two force-free and torque-free solid spheres $\left(\eta_{1}^{*}=\eta_{2}^{*} \rightarrow \infty\right.$ and rotation of particle takes place) are presented in Table 3 . The formulation of the collocation procedure for the motion of solid spheres in the undisturbed velocity field $\mathbf{v}_{\infty}(\mathbf{x})$ expressed by Eq. [2.1] is given by Appendix $\mathrm{A}$ and the dimensionless scalar function $C_{i}$ of particle $i$ is related to its angular velocity $\boldsymbol{\Omega}_{i}$ by

$$
\mathbf{\Omega}_{i}=\boldsymbol{\omega}+C_{i}(\mathbf{e} \times \mathbf{I}) \cdot \mathbf{E} \cdot \mathbf{e}, \quad i=1,2 .
$$

It is obvious that

$$
C_{1}(s, \lambda)=C_{2}\left(s, \lambda^{-1}\right),
$$


TABLE 2

The Scalar Functions $A, Q_{1}, P_{1}, Q_{2}$ and $P_{2}$ of Two $\mathrm{G}$ as Bubbles at Various Relative Radii and Spacings

\begin{tabular}{llccccc}
\hline$\lambda$ & \multicolumn{1}{c}{$2 / s$} & $A$ & $Q_{1}$ & $P_{1}$ & $Q_{2}$ & $P_{2}$ \\
\hline 1 & 0.2 & 0.0020 & -0.0010 & 0.0020 & & \\
& 0.4 & 0.0163 & -0.0079 & 0.0163 & & \\
& 0.6 & 0.0576 & -0.0261 & 0.0579 & & \\
& 0.8 & 0.1548 & -0.0518 & 0.1589 & & \\
& 0.9 & 0.2539 & -0.0777 & 0.2696 & $=Q_{1}$ & $=P_{1}$ \\
& 0.95 & 0.3415 & -0.0880 & 0.3765 & & \\
& 0.99 & 0.4935 & -0.0974 & 0.5854 & & \\
& 0.995 & 0.5411 & -0.0982 & 0.6571 & & \\
& 0.999 & 0.6245 & -0.0984 & 0.7833 & & \\
& 0.9999 & 0.7025 & -0.0985 & 0.9153 & & \\
& 1.0 & 1.0 & -0.0985 & 0.99 & & \\
2 & & & & & \\
& 0.2 & 0.0027 & -0.0024 & 0.0047 & -0.0003 & 0.0006 \\
& 0.4 & 0.0215 & -0.0189 & 0.0381 & -0.0023 & 0.0049 \\
& 0.6 & 0.0747 & -0.0632 & 0.1315 & -0.0074 & 0.0183 \\
& 0.8 & 0.1916 & -0.1454 & 0.3397 & -0.0155 & 0.0533 \\
& 0.9 & 0.3019 & -0.2006 & 0.5546 & -0.0199 & 0.0922 \\
& 0.95 & 0.3937 & -0.2308 & 0.7609 & -0.0219 & 0.1291 \\
& 0.99 & 0.5441 & -0.2558 & 1.1747 & -0.0234 & 0.1995 \\
& 0.995 & 0.5895 & -0.2589 & 1.3196 & -0.0236 & 0.2235 \\
& 0.999 & 0.6674 & -0.2615 & 1.5844 & -0.0237 & 0.2669 \\
& 0.9995 & 0.6925 & -0.2618 & 1.6747 & -0.0237 & 0.2809 \\
& 1.0 & 1.0 & -0.2621 & & -0.0237 & \\
5 & 0.2 & 0.0047 & -0.0046 & 0.0093 & -0.0000 & 0.0001 \\
5 & 0.4 & 0.0374 & -0.0370 & 0.0741 & -0.0003 & 0.0006 \\
& 0.6 & 0.1267 & -0.1247 & 0.2513 & -0.0009 & 0.0025 \\
& 0.8 & 0.3060 & -0.2935 & 0.6121 & -0.0016 & 0.0078 \\
& 0.9 & 0.4503 & -0.4131 & 0.9359 & -0.0019 & 0.0134 \\
& 0.95 & 0.5528 & -0.4804 & 1.2321 & -0.0019 & 0.0185 \\
& 0.99 & 0.6924 & -0.5366 & 1.8645 & -0.0019 & 0.0280 \\
& 0.995 & 0.7293 & -0.5437 & 2.1010 & -0.0019 & 0.0313 \\
& 0.999 & 0.7879 & -0.5494 & 2.5210 & -0.0019 & 0.0369 \\
1.0 & 1.0 & -0.5508 & & -0.0019 & \\
\hline & & & & & &
\end{tabular}

and

$$
C_{i} \rightarrow 0 \quad \text { as } \quad s \rightarrow \infty .
$$

The results in Table 3 agree very well with the far-field analytic expressions of the scalar functions obtained by Batchelor and Green (10) and with the near-field asymptotic formulas of these functions for two equally sized spheres obtained by Yoon and Kim (13). A comparison of our results of the functions $A, B$, and $C$ for two identical solid spheres $(\lambda=1)$ with those obtained by Lin et al. (9) using spherical bipolar coordinates shows a good agreement in the range of $2.0006 \leq s<\infty$. However, their numerical solutions of the functions $B$ and $C$ are in serious errors beyond this range (as $2<s<2.0006$ ).

Table 4 presents the collocation results of the scalar functions for two spheres of the same size $\left(a_{1}=a_{2}=a\right)$ having difference viscosities at various separations. The results in Tables 2-4 illustrate that both the relative-velocity functions $A$ and $B$ as well as the magnitudes of the stresslet functions $K_{1}, K_{2}$ and $P_{1}$ increase monotonically, for all values of $\eta_{1}^{*}, \eta_{2}^{*}$, and $\lambda$ (with $\lambda \geq 1$ ), with decreasing gap thickness between the two droplets (or increasing value of $2 / s)$. Indeed, $A \rightarrow 1$ as $2 / s \rightarrow 1$ in all cases, agreeing with the requirement of Eq. [2.6b]. On the other hand, the stresslet functions $Q_{1}, Q_{2}$, and $P_{2}$ are not necessarily varying with $2 / s$ monotonically for a given combination of $\eta_{1}^{*}, \eta_{2}^{*}$, and $\lambda$. Our results indicate that, for two droplets of the same viscosity $\left(\eta_{1}^{*}=\eta_{2}^{*}=\eta^{*}\right), A, B$, and the magnitudes of $K_{1}$ and $K_{2}$ increase with the increase of $\eta^{*}$ if the parameters $\lambda$ and $s$ are kept constant, and both $A$ and $B$ become larger when the size ratio $\lambda$ deviates more from unity for given values of $\eta^{*}$ and $s$, consistent with the results of Wang et al. (6). For two droplets of the same size $(\lambda=1), A, B$, and the magnitudes of $K_{1}$ and $K_{2}$ in general increase with the increase of $\eta_{1}^{*}$ (or $\eta_{2}^{*}$ ), keeping $\eta_{2}^{*}$ (or $\eta_{1}^{*}$ ) and $s$ constant. It can be seen in Table 3 that the scalar function $C$ of two equally sized solid spheres (with $C_{1}=C_{2}=C$ ) increases monotonically with the increase of $2 / s$; but for two solid spheres with $\lambda>1, C_{1}$ (of the smaller sphere) is not necessarily a monotonic function of $2 / s$. When the size ratio $\lambda$ of two droplets of the same fixed viscosity is increased, $C_{1}$ (in case $\eta^{*} \rightarrow \infty$ ) and the magnitude of $K_{1}$ become larger, while $C_{2}$ and the magnitude of $K_{2}$ turn smaller. In all cases, we have $0 \leq B<A \leq 1$ [which was found by Zinchenko (3) for the case of two identical droplets], $C_{i} \geq 0$, and $K_{i} \leq 0$.

\section{APPENDIX A: FORMULATION FOR THE MOTION OF TWO SOLID SPHERES}

For the slow motion of two solid spheres (i.e., fluid spheres with $\left.\eta_{1}^{*}=\eta_{2}^{*} \rightarrow \infty\right)$ in an unbounded fluid with an undisturbed velocity field $\mathbf{v}_{\infty}(\mathbf{x})$ as illustrated in Fig. 1, only the flow field of the surrounding fluid phase given by Eqs. [3.4a] and [3.5a] needs to be considered. The boundary conditions for the fluid velocity at the particle surfaces are

$$
r_{i}=a_{i}: \quad \mathbf{v}=\mathbf{U}_{i}+\boldsymbol{\Omega}_{i} \times a_{i} \mathbf{e}_{r i}, \quad i=1,2,
$$

instead of conditions [3.2a-c]. Here, $\boldsymbol{\Omega}_{i}$ is the angular velocity of particle $i$ and can be written as $\Omega_{i x} \mathbf{e}_{x}+$ $\Omega_{i y} \mathbf{e}_{y}+\Omega_{i z} \mathbf{e}_{z}$. The hydrodynamic torque experienced by the particle $i$ about its center can be calculated using Eq. [3.6] and is given by (19)

$$
\mathbf{T}_{i}=-8 \pi \eta \nabla\left[r_{i}^{3} \chi_{-2}^{(i)}\right]
$$

whereas the drag force on the particle is given by Eq. [3.7]. Substitution of Eq. [3.5a] into Eq. [A2] leads to

$$
\mathbf{T}_{i}=-8 \pi \eta\left(A_{i 11} \mathbf{e}_{x}+B_{i 11} \mathbf{e}_{y}+A_{i 01} \mathbf{e}_{z}\right) .
$$


TABLE 3

The Scalar Functions $A, B, C_{i}, K_{i}, Q_{i}$, and $P_{i}$ of Two Solid Spheres at Various Relative R adii and Spacings

\begin{tabular}{|c|c|c|c|c|c|c|c|c|c|c|c|}
\hline$\lambda$ & $2 / s$ & $A$ & $B$ & $C_{1}$ & $C_{2}$ & $K_{1}$ & $Q_{1}$ & $P_{1}$ & $K_{2}$ & $Q_{2}$ & $P_{2}$ \\
\hline \multirow[t]{12}{*}{1} & 0.2 & 0.0049 & 0.0001 & 0.0025 & \multirow{12}{*}{$=C_{1}$} & -0.0000 & -0.0024 & 0.0049 & \multirow{12}{*}{$=K_{1}$} & \multirow{12}{*}{$=Q_{1}$} & \multirow{12}{*}{$=P_{1}$} \\
\hline & 0.4 & 0.0390 & 0.0017 & 0.0196 & & -0.0006 & -0.0170 & 0.0378 & & & \\
\hline & 0.6 & 0.1336 & 0.0133 & 0.0636 & & -0.0046 & -0.0439 & 0.1245 & & & \\
\hline & 0.8 & 0.3447 & 0.0565 & 0.1451 & & -0.0181 & -0.0605 & 0.3125 & & & \\
\hline & 0.9 & 0.5562 & 0.1077 & 0.2130 & & -0.0306 & -0.0504 & 0.4961 & & & \\
\hline & 0.95 & 0.7226 & 0.1527 & 0.2671 & & -0.0385 & -0.0325 & 0.6468 & & & \\
\hline & 0.99 & 0.9272 & 0.2252 & 0.3543 & & -0.0454 & 0.0080 & 0.8395 & & & \\
\hline & 0.995 & 0.9618 & 0.2459 & 0.3804 & & -0.0463 & 0.0220 & 0.8730 & & & \\
\hline & 0.999 & 0.9920 & 0.2800 & 0.4245 & & -0.0470 & 0.0467 & 0.9025 & & & \\
\hline & 0.9999 & 0.9991 & 0.3094 & 0.4638 & & -0.0472 & 0.0694 & 0.9096 & & & \\
\hline & 0.99999 & 0.9998 & 0.3267 & 0.4871 & & -0.0472 & 0.0830 & 0.9103 & & & \\
\hline & 1.0 & 1.0 & 0.4060 & 0.5940 & & -0.0472 & 0.1454 & 0.9105 & & & \\
\hline \multirow[t]{11}{*}{2} & 0.2 & 0.0066 & 0.0001 & 0.0059 & 0.0007 & -0.0001 & -0.0058 & 0.0114 & -0.0000 & -0.0007 & 0.0015 \\
\hline & 0.4 & 0.0511 & 0.0022 & 0.0471 & 0.0057 & -0.0017 & -0.0404 & 0.0859 & -0.0002 & -0.0048 & 0.0116 \\
\hline & 0.6 & 0.1678 & 0.0172 & 0.1567 & 0.0177 & -0.0124 & -0.1044 & 0.2625 & -0.0015 & -0.0116 & 0.0396 \\
\hline & 0.8 & 0.4039 & 0.0746 & 0.3620 & 0.0403 & -0.0493 & -0.1347 & 0.5932 & -0.0060 & -0.0151 & 0.0947 \\
\hline & 0.9 & 0.6092 & 0.1424 & 0.5081 & 0.0645 & -0.0839 & -0.0773 & 0.9112 & -0.0100 & -0.0129 & 0.1367 \\
\hline & 0.95 & 0.7615 & 0.2014 & 0.5937 & 0.0900 & -0.1059 & 0.0126 & 1.1900 & -0.0124 & -0.0089 & 0.1645 \\
\hline & 0.99 & 0.9388 & 0.2963 & 0.6662 & 0.1450 & -0.1258 & 0.2253 & 1.5897 & -0.0144 & 0.0017 & 0.1928 \\
\hline & 0.995 & 0.9679 & 0.3240 & 0.6727 & 0.1651 & -0.1284 & 0.3036 & 1.6689 & -0.0147 & 0.0056 & 0.1963 \\
\hline & 0.999 & 0.9932 & 0.3709 & 0.6678 & 0.2032 & -0.1305 & 0.4510 & 1.7450 & -0.0149 & 0.0132 & 0.1988 \\
\hline & 0.9995 & 0.9966 & 0.3800 & 0.6626 & 0.2165 & -0.1308 & 0.5020 & 1.7567 & -0.0149 & 0.0162 & 0.1990 \\
\hline & 1.0 & 1.0 & $0.583^{*}$ & $0.417 *$ & $0.417 *$ & -0.1311 & & 1.7687 & -0.0149 & & 0.1992 \\
\hline \multirow[t]{10}{*}{5} & 0.2 & 0.0115 & 0.0001 & 0.0116 & 0.0001 & -0.0001 & -0.0110 & 0.0224 & -0.0000 & -0.0001 & 0.0002 \\
\hline & 0.4 & 0.0869 & 0.0044 & 0.0925 & 0.0007 & -0.0043 & -0.0754 & 0.1599 & -0.0000 & -0.0005 & 0.0015 \\
\hline & 0.6 & 0.2680 & 0.0339 & 0.3115 & 0.0020 & -0.0323 & -0.1808 & 0.4373 & -0.0002 & -0.0011 & 0.0050 \\
\hline & 0.8 & 0.5610 & 0.1451 & 0.7308 & 0.0049 & -0.1331 & -0.1716 & 0.7466 & -0.0009 & -0.0012 & 0.0094 \\
\hline & 0.9 & 0.7486 & 0.2704 & 1.0157 & 0.0090 & -0.2325 & 0.0306 & 0.9155 & -0.0014 & -0.0002 & 0.0098 \\
\hline & 0.95 & 0.8584 & 0.3718 & 1.0476 & 0.0140 & -0.2971 & 0.3106 & 1.0795 & -0.0017 & 0.0012 & 0.0090 \\
\hline & 0.99 & 0.9659 & 0.5184 & 1.1446 & 0.0263 & -0.3560 & 1.0014 & 1.4163 & -0.0018 & 0.0055 & 0.0073 \\
\hline & 0.995 & 0.9822 & 0.5583 & 1.0965 & 0.0311 & -0.3638 & 1.2702 & 1.5057 & -0.0019 & 0.0072 & 0.0068 \\
\hline & 0.999 & 0.9961 & 0.6240 & 0.9710 & 0.0405 & -0.3701 & 1.7972 & 1.6043 & -0.0019 & 0.0106 & 0.0062 \\
\hline & 1.0 & 1.0 & $0.910^{*}$ & $0.090 *$ & $0.090^{*}$ & -0.3716 & & & -0.0019 & & 0.0060 \\
\hline
\end{tabular}

* Values calculated using the numerical results obtained by Nir and Acrivos (12).

Because the particles are freely suspended in the fluid, no net torque from the fluid acts on the particles. From Eq. [A3], it is apparent that

$$
A_{i 01}=A_{i 11}=B_{i 11}=0, \quad i=1,2 .
$$

The translational and angular velocities $\mathbf{U}_{i}$ and $\boldsymbol{\Omega}_{i}$ of the two particles (12 components in total) can be determined by solving the 12 equations [A4] and [3.9] simultaneously.

When the undisturbed velocity field is the two-dimensional pure straining flow given by Eq. [3.10], the translational and angular velocities of the two solid spheres located on the $z$-axis are zero and the coefficients $A_{j m n}, D_{j m n}$, and $F_{j m n}$ in Eq. [3.5a] vanish. Application of the boundary condition (A1) with $\mathbf{U}_{i}=\boldsymbol{\Omega}_{i}=\mathbf{0}$ to the solution [3.4a] after the substitution of Eq. [3.10a] results in

$$
\begin{gathered}
\sum_{j=1}^{2} \sum_{n=2}^{\infty} G_{j i 2 n}^{(1)}\left(a_{i}, \mu_{i}\right)=-\kappa a_{i}\left(1-\mu_{i}^{2}\right), \\
\sum_{j=1}^{2} \sum_{n=2}^{\infty} G_{j i 2 n}^{(2)}\left(a_{i}, \mu_{i}\right)=-\kappa a_{i} \mu_{i}\left(1-\mu_{i}^{2}\right)^{1 / 2}, \\
\sum_{j=1}^{2} \sum_{n=2}^{\infty} G_{j i 2 n}^{(3)}\left(a_{i}, \mu_{i}\right)=\kappa a_{i}\left(1-\mu_{i}^{2}\right)^{1 / 2},
\end{gathered}
$$

where $i=1$ or 2 . In Eqs. [A5a-c], the functions $G_{j i m n}^{(l)}\left(r_{i}, \mu_{i}\right)$ with $m=0,1$, and 2 and $l=1,2$, and 3 are defined by Eq. [B7], and the dependence of $\phi$ factors out. Now, the boundary collocation method described in Section 3 can be used. The infinite series in boundary conditions $[\mathrm{A} 5 \mathrm{a}-\mathrm{c}]$ are trun- 
TABLE 4

The Scalar Functions $A, B, C_{i}, K_{i}, Q_{i}$, and $P_{i}$ of Two Equally Sized Droplets at Various R elative Viscosities and Spacings

\begin{tabular}{|c|c|c|c|c|c|c|c|c|c|c|c|}
\hline$\eta_{1}^{*}$ & $\eta_{2}^{*}$ & $2 / s$ & $A$ & $B$ & $C_{1}$ & $K_{1}$ & $Q_{1}$ & $P_{1}$ & $K_{2}$ & $Q_{2}$ & $P_{2}$ \\
\hline \multirow[t]{11}{*}{$\infty$} & 0 & 0.2 & 0.0035 & 0.0000 & 0.0010 & -0.0000 & -0.0010 & 0.0020 & -0.0000 & -0.0025 & 0.0050 \\
\hline & & 0.4 & 0.0279 & 0.0005 & 0.0078 & -0.0001 & -0.0073 & 0.0159 & -0.0003 & -0.0185 & 0.0388 \\
\hline & & 0.6 & 0.0966 & 0.0041 & 0.0250 & -0.0010 & -0.0215 & 0.0557 & -0.0023 & -0.0554 & 0.1303 \\
\hline & & 0.8 & 0.2555 & 0.0169 & 0.0532 & -0.0045 & -0.0422 & 0.1495 & -0.0089 & -0.1025 & 0.3411 \\
\hline & & 0.9 & 0.4220 & 0.0305 & 0.0727 & -0.0090 & -0.0578 & 0.2463 & -0.0143 & -0.1125 & 0.5906 \\
\hline & & 0.95 & 0.5773 & 0.0427 & 0.0905 & -0.0128 & -0.0703 & 0.3333 & -0.0172 & -0.0968 & 0.8662 \\
\hline & & 0.99 & 0.8460 & 0.0727 & 0.1426 & -0.0171 & -0.0845 & 0.4687 & -0.0193 & -0.0178 & 1.4555 \\
\hline & & 0.995 & 0.9115 & 0.0858 & 0.1672 & -0.0177 & -0.0860 & 0.4965 & -0.0195 & 0.0197 & 1.6238 \\
\hline & & 0.999 & 0.9794 & 0.1138 & 0.2207 & -0.0182 & -0.0857 & 0.5203 & -0.0197 & 0.0989 & 1.8145 \\
\hline & & 0.9999 & 0.9977 & 0.1426 & 0.2898 & -0.0183 & -0.0874 & 0.5244 & -0.0197 & 0.1596 & 1.8726 \\
\hline & & 1.0 & 1.0 & 0.16 & 0.37 & -0.0183 & -0.11 & 0.526 & -0.0197 & 0.29 & 1.89 \\
\hline \multirow[t]{11}{*}{$\infty$} & 1 & 0.2 & 0.0042 & 0.0000 & 0.0017 & -0.0000 & -0.0017 & 0.0034 & -0.0000 & -0.0024 & 0.0049 \\
\hline & & 0.4 & 0.0334 & 0.0012 & 0.0137 & -0.0004 & -0.0122 & 0.0268 & -0.0005 & -0.0175 & 0.0379 \\
\hline & & 0.6 & 0.1144 & 0.0089 & 0.0442 & -0.0029 & -0.0329 & 0.0899 & -0.0040 & -0.0476 & 0.1242 \\
\hline & & 0.8 & 0.2980 & 0.0374 & 0.0979 & -0.0119 & -0.0529 & 0.2285 & -0.0158 & -0.0735 & 0.3104 \\
\hline & & 0.9 & 0.4802 & 0.0678 & 0.1395 & -0.0213 & -0.0599 & 0.3633 & -0.0268 & -0.0699 & 0.5020 \\
\hline & & 0.95 & 0.6351 & 0.0914 & 0.1725 & -0.0280 & -0.0641 & 0.4767 & -0.0337 & -0.0553 & 0.6802 \\
\hline & & 0.99 & 0.8674 & 0.1248 & 0.2339 & -0.0344 & -0.0700 & 0.6412 & -0.0397 & -0.0144 & 0.9907 \\
\hline & & 0.995 & 0.9207 & 0.1346 & 0.2563 & -0.0353 & -0.0710 & 0.6762 & -0.0405 & 0.0019 & 1.0730 \\
\hline & & 0.999 & 0.9791 & 0.1527 & 0.3011 & -0.0360 & -0.0713 & 0.7113 & -0.0411 & 0.0350 & 1.1723 \\
\hline & & 0.9999 & 0.9972 & 0.1711 & 0.3391 & -0.0361 & -0.0672 & 0.7202 & -0.0413 & 0.0650 & 1.2067 \\
\hline & & 1.0 & 1.0 & 0.18 & 0.36 & -0.0361 & -0.066 & 0.721 & -0.0413 & 0.075 & 1.22 \\
\hline \multirow[t]{11}{*}{0} & 1 & 0.2 & 0.0027 & 0.0000 & & -0.0000 & -0.0017 & 0.0035 & -0.0000 & -0.0010 & 0.0020 \\
\hline & & 0.4 & 0.0220 & 0.0003 & & -0.0002 & -0.0132 & 0.0275 & -0.0001 & -0.0075 & 0.0159 \\
\hline & & 0.6 & 0.0768 & 0.0022 & & -0.0012 & -0.0408 & 0.0940 & -0.0007 & -0.0230 & 0.0555 \\
\hline & & 0.8 & 0.2036 & 0.0089 & & -0.0047 & -0.0806 & 0.2483 & -0.0032 & -0.0467 & 0.1481 \\
\hline & & 0.9 & 0.3339 & 0.0156 & & -0.0079 & -0.0972 & 0.4227 & -0.0062 & -0.0624 & 0.2450 \\
\hline & & 0.95 & 0.4526 & 0.0206 & & -0.0098 & -0.0988 & 0.6033 & -0.0088 & -0.0730 & 0.3356 \\
\hline & & 0.99 & 0.6716 & 0.0271 & & -0.0115 & -0.0874 & 0.9932 & -0.0117 & -0.0851 & 0.5115 \\
\hline & & 0.995 & 0.7427 & 0.0285 & & -0.0118 & -0.0834 & 1.1328 & -0.0122 & -0.0872 & 0.5714 \\
\hline & & 0.999 & 0.8618 & 0.0299 & & -0.0119 & -0.0785 & 1.3762 & -0.0126 & -0.0891 & 0.6746 \\
\hline & & 0.9999 & 0.9477 & 0.0304 & & -0.0120 & -0.0769 & 1.5603 & -0.0126 & -0.0897 & 0.7505 \\
\hline & & 1.0 & 1.0 & 0.0304 & & -0.0120 & -0.0766 & 1.61 & -0.0126 & -0.0899 & 0.77 \\
\hline
\end{tabular}

cated after $N$ terms, and these truncated conditions are applied at $N$ discrete values of $\theta_{i}$ along the particle surfaces. This generates a system of $6 \mathrm{~N}$ linear algebraic equations for the $6 N$ unknown coefficients $B_{j 2 n}, C_{j 2 n}$, and $E_{j 2 n}$ (all coefficients with $m \neq 2$ disappear) in Eq. [3.5a]. After these coefficients are solved by a matrix reduction technique, the scalar functions $K_{i}$ for given values of $s$ and $\lambda$ can be obtained by comparing Eqs. [3.11] and [3.13].

When the undisturbed velocity field is the unidirectional shear flow expressed by Eq. [3.14], the particle velocities $\mathbf{U}_{i}=$ $U_{i x} \mathbf{e}_{x}$ and $\boldsymbol{\Omega}_{i}=\Omega_{i y} \mathbf{e}_{y}$. Substituting Eqs. [3.14b, c] into Eq. [4.1], we have

$$
\Omega_{i y}=\left(1+C_{i}\right) \kappa .
$$

Application of Eq. [A1] with $U_{i y}=U_{i z}=\Omega_{i x}=\Omega_{i z}=0$ to Eq. [3.4a] after the substitution of Eq. [3.14a] yields

$$
\begin{aligned}
& \sum_{j=1}^{2} \sum_{n=1}^{\infty} G_{j i 1 n}^{(1)}\left(a_{i}, \mu_{i}\right) \\
& \quad=\left[U_{i x}-2(i-1) \kappa r-2 \kappa a_{i} \mu_{i}\right]\left(1-\mu_{i}^{2}\right)^{1 / 2}, \\
& \sum_{j=1}^{2} \sum_{n=1}^{\infty} G_{j i 1 n}^{(2)}\left(a_{i}, \mu_{i}\right) \\
& \quad=\left[U_{i x}-2(i-1) \kappa r-2 \kappa a_{i} \mu_{i}\right] \mu_{i}+a_{i} \Omega_{i y},
\end{aligned}
$$

$$
\begin{aligned}
& \sum_{j=1}^{2} \sum_{n=1}^{\infty} G_{j i 1 n}^{(3)}\left(a_{i}, \mu_{i}\right) \\
& \quad=-U_{i x}+2(i-1) \kappa r+2 \kappa a_{i} \mu_{i}-a_{i} \Omega_{i y} \mu_{i},
\end{aligned}
$$

where $i=1$ or 2 . Using the boundary collocation technique, we 
can apply the truncated form of Eqs. [A7a-c] at $N$ discrete values of $\theta_{i}$ along the surface of each sphere and generate a set of $6 \mathrm{~N}$ linear algebraic equations for the equal number of unknown coefficients $B_{j 1 n}, C_{j 1 n}$, and $E_{j 1 n}$ (coefficients $A_{j m n}$, $D_{j m n}, F_{j m n}$ and the others with $m \neq 1$ are equal to zero) in Eq. [3.5a]. After these coefficients are determined, the translational velocity, angular velocity, and force dipole strength of each particle can be calculated, and a comparison of these results with those given by Eqs. [3.15], [A6], and [3.16] leads to the scalar functions $B, C_{i}$, and $Q_{i}$ for given values of $s$ and $\lambda$.

When the undisturbed velocity field is the axisymmetric pure straining flow given by Eq. [3.19], the angular velocities of the particles are zero and the translational velocities $\mathbf{U}_{i}=U_{i z} \mathbf{e}_{z}$. Application of Eq. [A1] with $U_{i x}=U_{i y}=0$ and $\boldsymbol{\Omega}_{i}=\mathbf{0}$ to Eq. [3.4a] after the substitution of Eq. [3.19a] leads to

$$
\begin{aligned}
\sum_{j=1}^{2} \sum_{n=1}^{\infty} G_{j i 0 n}^{(1)} & \left(a_{i}, \mu_{i}\right) \\
& =\left[U_{i z}-(i-1) \kappa r\right] \mu_{i}+\frac{1}{2} \kappa a_{i}\left(1-3 \mu_{i}^{2}\right)
\end{aligned}
$$

$$
\begin{aligned}
\sum_{j=1}^{2} \sum_{n=1}^{\infty} G_{j i 0 n}^{(2)}\left(a_{i},\right. & \left.\mu_{i}\right) \\
& =\left[(i-1) \kappa r+\frac{3}{2} \kappa a_{i} \mu_{i}\right]\left(1-\mu_{i}^{2}\right)^{1 / 2},
\end{aligned}
$$

where $i=1$ or 2 . The first $N$ sets of the coefficients $C_{j 0 n}$ and $E_{f 0 n}$ (coefficients $A_{j m n}, B_{j m n}, D_{j m n}$, and $F_{j m n}$ and the others with $m \neq 0$ are all zero) in Eq. [3.5a] can be solved using Eqs. [A8a, b] and the boundary collocation technique. The scalar functions $A$ and $P_{i}$ for given values of $s$ and $\lambda$ are to be determined through the same procedure as that described in Subsection 3.3.

For the particular case of the simultaneous motion of one fluid sphere and one solid sphere in the undisturbed velocity field $\mathbf{v}_{\infty}(\mathbf{x})$ expressed by Eq. [2.1], the formulation of the collocation procedure is a combination of the above description in this appendix and that given by Section 3 .

\section{APPENDIX B: DEFINITION OF SOME FUNCTIONS IN SECTION 3 AND APPENDIX A}

For conciseness, the definitions of the functions $H_{i m}^{(k)}\left(\mu_{i}\right)$ and $G_{j i m n}^{(l)}\left(r_{i}, \mu_{i}\right)$ with $i=1$ and $2, j=1$ and $2, m=0,1$, and $2, k=1,2, \ldots, 6$, and $l=1,2, \ldots, 8$ in Section 3 and Appendix $\mathrm{A}$ are listed in this appendix. With a saving in printing space, these lengthy formulas are not reproduced here. The full version of this appendix is available on request from the authors.

\section{ACKNOWLEDGMENT}

This work was supported by the National Science Council of the Republic of China under Grant NSC 87-2214-E002-005.

\section{REFERENCES}

1. Batchelor, G. K., and Green, J. T., J. Fluid Mech. 56, 401 (1972).

2. Batchelor, G. K., J. Fluid Mech. 83, 97 (1977).

3. Zinchenko, A. Z., PMM Applied Math. Mech. 48, 198 (1984).

4. Zeichner, G. R., and Schowalter, W. R., AIChE J. 23, 243 (1977).

5. Alder, P. M., J. Colloid Interface Sci. 83, 106 (1981).

6. Wang, H., Zinchenko, A. Z., and Davis, R. H., J. Fluid Mech. 265, 161 (1994).

7. Acrivos, A., Batchelor, G. K., Hinch, E. J., Koch, D. L., and Mauri, R., J. Fluid Mech. 40, 651 (1992).

8. Wang, Y., Mauri, R., and Acrivos, A., J. Fluid Mech. 327, 255 (1996).

9. Lin, C. J., Lee, K. J., and Sather, N. F., J. Fluid Mech. 43, 35 (1970).

10. Batchelor, G. K., and Green, J. T., J. Fluid Mech. 56, 375 (1972).

11. Wakiya, S., J. Phys. Soc. Japan 31, 1581 (1971).

12. Nir, A., and Acrivos, A., J. Fluid Mech. 59, 209 (1973).

13. Yoon, B. J., and Kim, S., J. Fluid Mech. 185, 437 (1987).

14. Ganatos, P., Pfeffer, R., and Weinbaum, S., J. Fluid Mech. 84, 79 (1978).

15. Hassonjee, Q., Pfeffer, R., and Ganatos, P., Int. J. Multiphase Flow 18, 353 (1992).

16. Zinchenko, A. Z., PMM Applied Math. Mech. 47, 37 (1984).

17. Keh, H. J., and Tseng, Y. K., AIChE J. 38, 1881 (1992).

18. Lamb, H., "Hydrodynamics,", 6th ed. Dover, New York, 1945.

19. Happel, J., and Brenner, H., "Low Reynolds Number Hydrodynamics,' Martinus Nijhoff, Dordrecht, The Netherlands, 1983.

20. Gerald, C. F., and Wheatley, P. O., "Applied Numerical Analysis," 5th ed. Addison-Wesley, Reading, MA, 1994. 